02,12

\title{
Флуктуационное образование фазовых солитонов в двузонных сверхпроводящих мостиках
}

\author{
(C) П.М. Марычев \\ Институт фоизики микроструктур РАН, \\ Нижний Новгород, Россия \\ E-mail: marychevpm@ipmras.ru
}

На основе теории Гинзбурга-Ландау была рассчитана зависимость пороговой энергии образования центра проскальзывания фазы $\delta F_{\text {thr }}$ от тока для двузонного сверхпроводящего мостика. Обнаружено, что в случае достаточно длинных мостиков и слабой межзонной джозефсоновской связи существует область токов, при которых возможно флуктуационное переключение из состояния с нулевой межзонной разностью фаз в состояния с фазовым солитоном, что отражается в значительном уменьшении пороговой энергии $\delta F_{\mathrm{thr}}$, a также флуктуационное изменение числа фазовых солитонов в мостике. В случае сильной межзонной связи флуктуационное образование фазовых солитонов отсутствует, и зависимость $\delta F_{\text {thr }}(I)$ стремится к выражению, полученному ранее Лангером и Амбегаокаром для однозонного сверхпроводника.

Работа выполнена при поддержке гранта Российского фонда фундаментальных исследований \# 17-12-01383 (раздел 3) и Фонда развития теоретической физики „Базис“ (раздел 4).

DOI: $10.21883 /$ FTT.2018.11.46648.16NN

\section{1. Введение}

Характерной особенностью сверхпроводящих квазиодномерных каналов (примером которых являются узкие сверхпроводящие мостики) является существование резистивного состояния, в котором в канале сосуществуют сверхпроводимость и электрическое поле [1]. Подобное состояние определяется возникновением в канале центров проскальзывания фазы (ЦПФ) - точек в канале, в которых модуль параметра порядка обращается в ноль, а его фаза претерпевает скачок на кратную $2 \pi$ величину. Основными механизмами возникновения ЦПФ являются тепловые или квантовые флуктуации в системе, приводящие к наличию конечного сопротивления ниже критической температуры сверхпроводящего перехода $T_{c}$, и спонтанное возбуждение под действием тока. Токовое образование ЦПФ, в отличие от флуктуационного, происходит только при достаточно большом токе, чтобы привести к неустойчивости сверхпроводящего состояния в какой-либо из точек канала. В случае мостика со сверхпроводящими берегами этот ток соответствует критическому току Гинзбурга-Ландау. Флуктуационный механизм был впервые рассмотрен для случая длинных сверхпроводящих мостиков в работе [2]. Согласно этой работе, вероятность возникновения ЦПФ характеризуется энергетическим барьером $\delta F_{\mathrm{thr}}$, который необходимо преодолеть системе под действием флуктуации. В рамках теории Гинзбурга-Ландау была получена зависимость величины барьера $\delta F_{\text {thr }}$ от тока через мостик, которая в дальнейшем была обобщена на случай мостиков произвольной длины [3].

Флуктуационное образование ЦПФ ранее исследовалось в сверхпроводниках с одним параметром порядка. В 2001 году были открыты многозонные сверхпроводники [4], в которых возможно существование двух и более сверхпроводящих параметров порядка $\Psi_{k}=\Delta_{k} \exp \left(i \theta_{k}\right)$.
Многозонная природа сверхпроводимости в этих сверхпроводниках приводит к появлению новых физических эффектов, отсутствующих в однозонных сверхпроводниках. Одним из таких эффектов являются фазовые солитоны - пространственно-неоднородное распределение межзонной разности фаз $\theta_{i}-\theta_{j}$, меняющееся на протяжении солитона на $2 \pi$. Пример распределения фаз параметров порядка в различных зонах в сверхпроводящем мостике, соответствующего наличию фазового солитона, представлен на рис. 1. Фазовые солитоны были предсказаны в работе Танаки [5], и свидетельства их существования были обнаружены в искусственных двузонных сверхпроводящих структурах в работах [6] и [7]. Поскольку основное состояние многозонного сверхпроводника является, как правило, синфазным (т.е. $\theta_{i}-\theta_{j}=0$ или $\pi$ ), были предложены различные методы по созданию фазовых солитонов в сверхпроводниках. В работе Гуревича и Винокура [8] было предложено генерировать фазовые солитоны в двузонной сверхпроводящей системе через проскальзывания фазы, возникающие при превышении критического сверхтока „слабой“ зоны (зоны с меньшей амплитудой сверхпроводящего параметра порядка). В работе [9] было рассмотрено влияние на токовый механизм генерации фазовых солитонов длины сверхпроводящего мостика и силы межзонной связи и отмечено, что он работает только при достаточно больших длинах и весьма слабой межзонной джозефсоновской связи.

В однозонных сверхпроводящих мостиках флуктуационное возникновение ЦПФ приводит либо к релаксации к исходному стационарному сверхпроводящему состоянию, либо, при достаточно большом токе, система нагревается и переходит в резистивное состояние. Однако, наличие двух параметров порядка и, соответственно, солитонных состояний в двузонных сверхпроводниках делает возможным переход системы после флуктуационного 


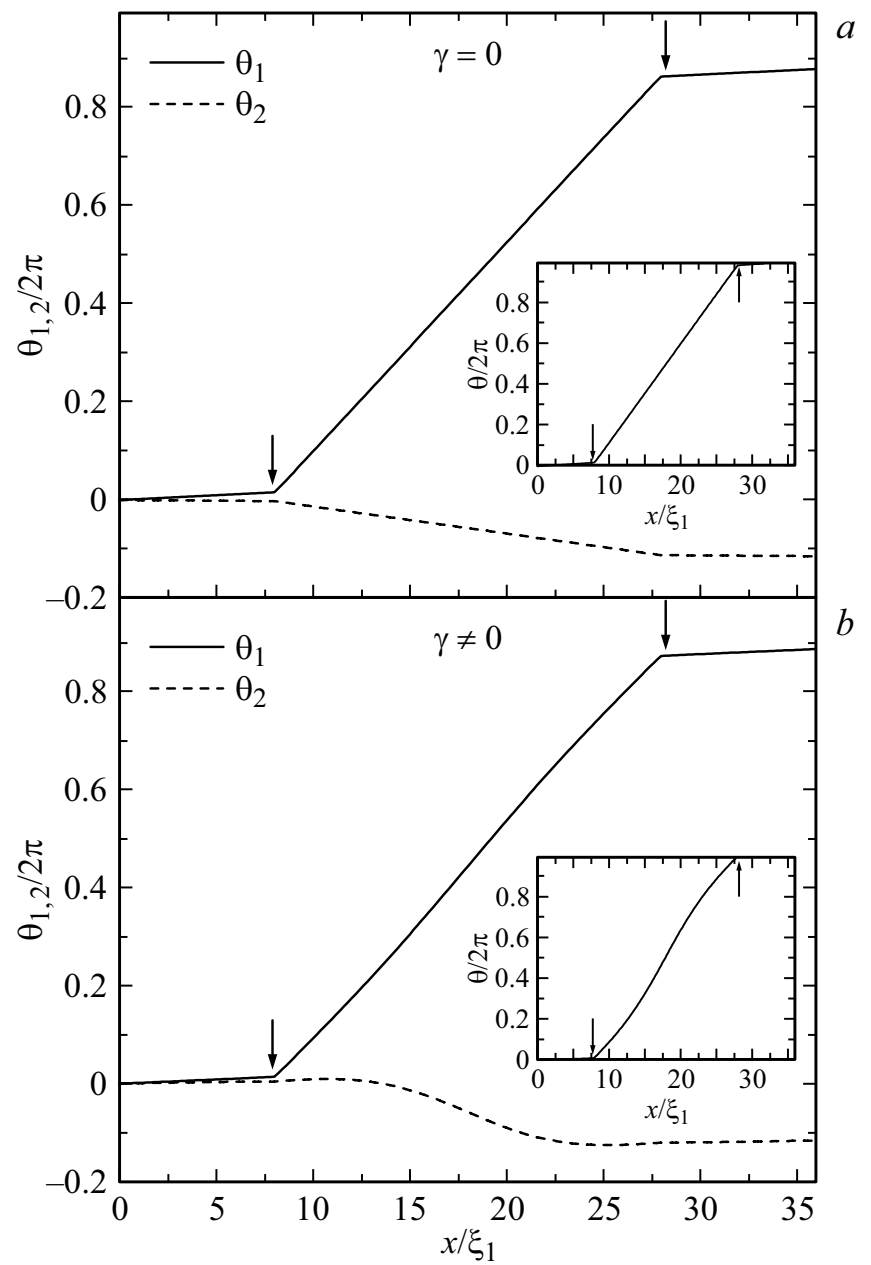

Рис. 1. Пространственное распределение фаз параметров порядков различных зон в сверхпроводящем мостике для случаев нулевой $(a)$ и ненулевой $(b)$ межзонной джозефсоновской связи $\gamma$. На вставках показано пространственное распределение межзонной разности фаз $\theta$. Стрелками указаны границы мостик/берег.

проскальзывания фазы на $2 \pi$ в состояние с фазовым солитоном вместо исходного. В связи с этим представляет интерес изучение нового, флуктуационного механизма создания состояний с фазовыми солитонами и влияния длины мостика и силы межзонного взаимодействия на возможность реализации такого механизма. Поскольку для перехода в солитонное состояние, в отличие от перехода в синфазное состояние, требуется возникновение ЦПФ только в одной (слабой) зоне, возможность такого перехода может повлиять на форму токовой зависимости энергии возникновения ЦПФ. В настоящей работе на основе одномерной нестационарной теории Гинзбурга-Ландау проводится анализ влияния длины мостика и силы межзонного джозефсоновского взаимодействия на токовую зависимость пороговой энергии образования ЦПФ $\delta F_{\mathrm{thr}}(I)$ в двузонных сверхпроводящих мостиках и отличий токовой зависимости $\delta F_{\mathrm{thr}}(I)$ от однозонного случая. Помимо двузонных сверхпровод- ников, в качестве материала мостика можно использовать двухслойную структуру из тонких сверхпроводящих пленок со слабой связью, как это было предложено в работе [8]. Было обнаружено, что в случае слабой межзонной связи и длине мостика $L \gg \xi_{1}\left(\xi_{1}-\right.$ длина когерентности в сильной зоне) в двузонных сверхпроводящих мостиках возможно флуктуационное образование фазовых солитонов при токах, превышающих определенное значение $j_{s}$, уменьшающееся с ростом длины мостика. Таким образом, возможно существование фазовых солитонов в системе при токах, недостаточных для токового механизма генерации, предложенного в [8]. Ток $j_{s}$ соответствует нижней границе области токов, при которой солитонное состояние становится энергетически выгоднее синфазного. При токе $j<j_{s}$ система, аналогично однозонному случаю, переходит в исходное состояние, которое является синфазным, и поэтому ЦПФ возникают парно в обеих зонах. Поскольку для переключения в солитонное состояние достаточно возникновения ЦПФ только в слабой зоне, что требует значительно меньше энергии, чем парное возникновение ЦПФ, в области токов вблизи $j_{s}$ происходит быстрое падение $\delta F_{\text {thr }}$, сглаживающееся с ростом межзонной связи. Кроме того, при длинах мостика, достаточно больших для устойчивости многосолитонных состояний, присутствуют скачки $\delta F_{\text {thr }}(I)$, соответствующие индуцированному током изменению числа фазовых солитонов в системе. При достаточно большой силе межзонной связи эти особенности исчезают и зависимость $\delta F_{\mathrm{thr}}(I)$ приближается к виду, характерному для длинных однозонных сверхпроводящих мостиков.

\section{2. Метод}

В данной работе рассматривается система, состоящая из сверхпроводящего мостика с площадью сечения $S$ и длиной $L$, соединяющего два сверхпроводящих берега с площадью сечения $S_{\text {pad. Предполагается, что }}$ система находится при температуре $T<T_{c 2}<T_{c 1}$, где $T_{c 1}$ и $T_{c 2}$ - критические температуры в отсутствие межзонной связи сильной и слабой зон соответственно. Считая, что максимальный поперечный размер системы $d_{\text {pad }} \sim \sqrt{S_{\text {раd }}} \ll \xi_{1}$, задача сводится к одномерной и учитывается зависимость только от продольной координаты $x$. Для расчета пороговой энергии образования ЦПФ $\delta F_{\mathrm{thr}}$ численно решались одномерные нестационарные уравнения Гинзбурга-Ландау [10], обобщенные на случай двузонной сверхпроводимости [11], вместе с уравнением на полную плотность тока $j$ в мостике. После обезразмеривания эти уравнения принимают вид

$$
\begin{gathered}
u_{1}\left(\frac{\partial}{\partial t}+i \varphi\right) \Psi_{1}-\Psi_{1}+\left|\Psi_{1}\right|^{2} \Psi_{1}-\frac{\partial^{2} \Psi_{1}}{\partial x^{2}}-\gamma \Psi_{2}=0 \\
u_{2}\left(\frac{\partial}{\partial t}+i \varphi\right) \Psi_{2}-\alpha \Psi_{2}+\beta\left|\Psi_{2}\right|^{2} \Psi_{2}-g \frac{\partial^{2} \Psi_{2}}{\partial x^{2}}-\gamma \Psi_{1}=0,
\end{gathered}
$$




$$
j=-(1+\sigma) \frac{\partial \varphi}{\partial x}+\operatorname{Im}\left(\Psi_{1}^{*} \frac{\partial \Psi_{1}}{\partial x}\right)+g \operatorname{Im}\left(\Psi_{2}^{*} \frac{\partial \Psi_{2}}{\partial x}\right),
$$

где $\alpha=\left|\alpha_{2}\right| /\left|\alpha_{1}\right|, \beta=\beta_{2} / \beta_{1}, g=g_{2} / g_{1}$ и $\sigma=\sigma_{n 2} / \sigma_{n 1}$. $\varphi$ - это электрический потенциал, $\sigma_{n k}-$ нормальная проводимость в зоне $k(k=1,2)$. $\alpha_{k}, \beta_{k}$ и $g_{k}$ - коэффициенты разложения в функционале Гинзбурга-Ландау, a $\gamma$ - константа межзонного джозефсоновского взаимодействия. Выражения для $\alpha_{k}, \beta_{k}, g_{k}$ и $\gamma$ через микроскопические параметры были получены в работах [12] и [13] для чистого и грязного случая соответственно. Координата $x$ выражена в единицах длины когерентности сильной зоны $\xi_{1}=\sqrt{g_{1} \hbar^{2} /\left|\alpha_{1}\right|}$, параметры порядка выражены в единицах однородного, безтокового значения модуля параметра порядка в сильной зоне $\left|\Psi_{10}\right|=\sqrt{\left|\alpha_{1}\right| / \beta_{1}}$, время обезразмерено на время релаксации тока в сильной зоне $t_{1}=\beta_{1} \sigma_{n 1} / 8 e^{2} g_{1}\left|\alpha_{1}\right|$, константа межзонного джозефсоновского взаимодействия $\gamma$ обезразмерена на $\left|\alpha_{1}\right|$, а плотность тока обезразмерена на $j_{0}=4 e \hbar g_{1}\left|\Psi_{10}\right|^{2} / \xi_{1}$. Сверхтекучий импульс каждой из зон $q_{k}=\hbar \nabla \theta_{k}$ выражен в единицах $\hbar / \xi_{1}$. В синфазном состоянии межзонная разность фаз $\theta=\theta_{1}-\theta_{2}$ определяется знаком константы межзонной связи $\gamma$, и для $\gamma$ положительной $\theta=0$, а для отрицательной $\theta=\pi$. В дальнейшем мы будем считать, что $\gamma>0$. Параметры $u_{k}$ в уравнениях (1), (2) характеризуют отношение времен релаксации параметров порядка в соответствующей зоне к времени релаксации тока первой, сильной зоны. В расчетах для простоты предполагалось, что $u_{1}=u_{2}=u$.

Предполагалось, что площадь сечения сверхпроводящих берегов намного больше площади сечения мостика, что обеспечивает близкую к нулевой плотность тока и равновесное сверхпроводящее состояние в берегах. В этом случае уравнения (1), (2) решаются с граничными условиями

$$
\begin{aligned}
& \Psi_{k}(0, t+\Delta t)=\Psi_{k}(0, t)=\left|\Psi_{k}^{0}\right|, \\
& \Psi_{k}(L, t+\Delta t)=\Psi_{k}(L, t) e^{-i \varphi_{L} \Delta t},
\end{aligned}
$$

где $\Psi_{k}^{0}$ - значение параметра порядка в берегах при нулевом токе, $L$ - длина мостика, $\varphi_{L}-$ значение электрического потенциала в точке $x=L$, а $\Delta t-$ шаг по времени. Чтобы найти электрический потенциал $\varphi$, уравнение (3) решалось на каждом шаге по времени при заданном полном токе и граничном условии $\varphi(0)=0$. Критерием достижения стационарного состояния являлось условие $\varphi(L)-\varphi(0)=0$. В случае малых длин мостика $L$ для учета подавления параметров порядка в берегах использовались граничные условия в глубине берегов $\left(x=L_{\mathrm{sys}}=L+16 \xi_{1}\right)$, дополненные условиями на границе мостик/берег

$$
\begin{aligned}
& \left.\frac{d \Psi_{1,2}^{L}}{d x}\right|_{\frac{L_{\mathrm{sys}}-L}{2}}=\left.\frac{S_{\mathrm{pad}}}{S} \frac{d \Psi_{1,2}^{C}}{d x}\right|_{\frac{L_{\mathrm{sys}}-L}{2}}, \\
& \left.\frac{S_{\mathrm{pad}}}{S} \frac{d \Psi_{1,2}^{C}}{d x}\right|_{\frac{L_{\mathrm{sys}+L}}{2}}=\left.\frac{d \Psi_{1,2}^{R}}{d x}\right|_{\frac{L_{\mathrm{sys}+L}}{2}},
\end{aligned}
$$

Эти условия возникают из варьирования функционала Гинзбурга-Ландау для сверхпроводника с зависящим от координаты $x$ сечением [3].
Для определения пороговой энергии образования ЦПФ использовалось выражение

$$
\delta F_{\mathrm{thr}}=F_{\text {saddle }}-F_{\text {ground }}-\frac{\hbar}{2 e} I \delta \theta_{k},
$$

где $I$ - это полный сверхток в мостике, $\delta \theta_{k}-$ дополнительная разность фаз между концами мостика, возникающая в седловом состоянии, а $F_{\text {saddle }}$ и $F_{\text {ground }}-$ свободные энергии седлового и основного состояний соответственно. В случае наличия в основном состоянии фазовых солитонов использовалась (аналогично работе [14], где вместо различных зон рассматривались различные нанопроволоки, соединяющие массивные контакты) разность фаз между концами в той зоне, в которой не происходило проскальзывания фазы, приведшего к образованию фазового солитона. Свободная энергия определялась как

$$
F_{\text {saddle, ground }}=-\frac{S \beta_{1}}{2} \int\left|\Psi_{1}\right|^{4} d x-\frac{S \beta_{2}}{2} \int\left|\Psi_{2}\right|^{4} d x .
$$

В вычислениях и в представленных ниже результатах энергия выражалась в единицах $F_{0}=\hbar^{2} g_{1}\left|\Psi_{10}\right|^{2} \xi_{1}^{3}$.

Уравнения (1)-(3) решались численно. Для поиска седлового состояния использовался численный метод, аналогичный предложенному в [15]: в центре мостика при заданном значении полного тока фиксировался модуль параметра порядка в сильной или слабой зоне, либо в обеих зонах одновременно. После нахождения соответствующих такой фиксации распределений параметров порядка, система релаксировала уже со свободными значениями параметров порядка в центре. Минимальное значение $\left|\Psi_{2}\right|$ в центре при фиксированном $\left|\Psi_{1}\right|$ (или минимальное значение $\left|\Psi_{1,2}\right|$ при фиксации только одного параметра порядка), которое не приводит при релаксации к возникновению ЦПФ, соответствует седловому состоянию системы. Далее из полученного набора седловых состояний, соответствующих различным $\left|\Psi_{1}\right|$ в центре, выбирается интересующее нас минимальное состояние.

\section{3. Пороговая энергия образования ЦПФ в системе без межзонной джозефсоновской связи}

Вначале рассмотрим случай, когда константа межзонного джозефсоновского взаимодействия $\gamma=0$. Подобная система с двумя параметрами порядка может соответствовать жидкому металлическому водороду [16], либо сверхпроводнику с прененебрежимо малой межзонной связью $\gamma \ll \alpha$. Чтобы уменьшить число свободных параметров системы, примем $\beta=g=1$ и будем менять только величину параметра $\alpha$.

При малых токах основным состоянием системы является синфазное состояние, в котором межзонная разность фаз $\theta=0$, и сверхтекучий импульс $q^{\mathrm{pl}}$ в этом состоянии одинаков для обеих зон. В длинном 


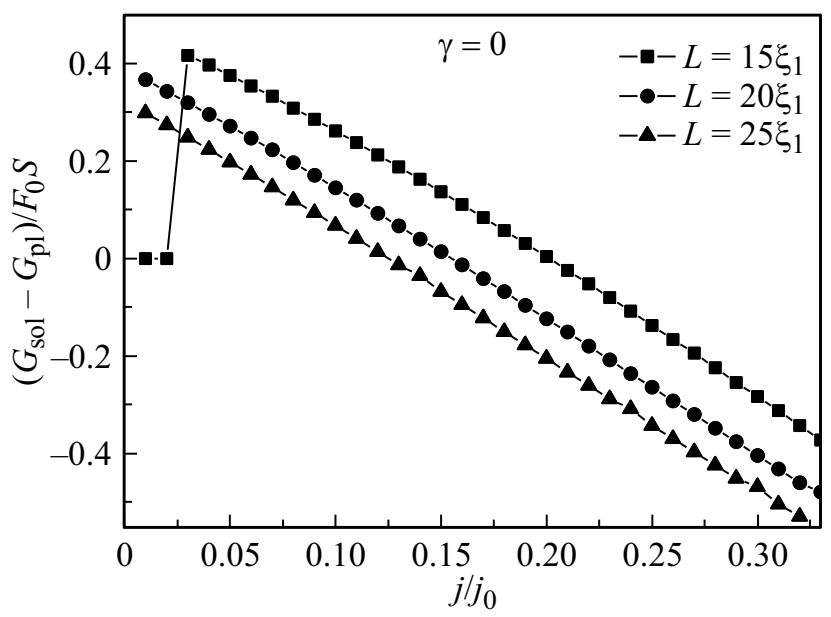

Рис. 2. Зависимость энергии Гиббса односолитонного состояния $G_{\text {sol }}$ односительно энергии Гиббса синфазного состояния $G_{\mathrm{pl}}$ от плотности полного сверхтока $j$ в мостике при различных длинах мостика $L$.

мостике подобное состояние обладает собственным критическим импульсом $q_{c}^{\mathrm{pl}}$. В случае разных зон $\alpha<1$, и тогда $q_{c}^{\mathrm{pl}}$ превышает критический импульс слабой зоны $q_{c 2}=\sqrt{\alpha / 3}$. Как было показано в работе [9], превышение $q_{c 2}$ приводит к перераспределению импульсов между зонами и образованию стационарного состояния с неоднородной солитоноподобной межзонной разностью фаз $\theta=2 \pi m x / L$, где $m$-целое число, равное разности количества проскальзываний фаз в сильной и слабой зонах, а также числу солитонов в мостике. Число солитонов в мостике меняется с увеличением полного сверхтока $I$ и длины мостика $L$. Солитоноподобные состояния существуют только при достаточно больших длинах, и при длинах мостика $L \lesssim 10-20 \xi_{1}$ эти состояния отсутствуют.

Несмотря на то, что солитоноподобные состояния образуются при токах, соответствующих импульсу $q_{c 2}$, они могут быть энергетически выгоднее синфазных состояний при меньших токах, на что указывал еще Гуревич в своей работе [8]. Поскольку в исследуемой системе является фиксированным полный сверхток, для сравнения энергии этих состояний были рассчитаны энергии Гиббса $G=F-2 I \Delta \theta_{k}$ для состояний с солитонами и без них. Здесь $\Delta \theta_{k}-$ набег фазы параметра порядка между концами мостика. В случае синфазного состояния он одинаковый для каждой из зон, в случае солитоноподобных состояний для однозначности учитывается набег фазы в зоне, в которой не происходило проскальзываний фазы. На рис. 2 представлено сравнение численно рассчитанных токовых зависимостей энергий Гиббса с солитоном и без солитона. Расчеты производились для системы, характеризуемой отношением собственных критических токов зон $R=j_{c 2} / j_{c 1}=0.15$. В этом случае для представленных длин значение плотности сверхтока $j\left(q_{c 2}\right)$, приводящего к образованию ЦПФ, составляет $\sim 0.33 j_{0}$. Видно, что состояние с солитоном действительно становится энергетически выгоднее синфазного состояния при токе $j_{s}$, меньшем $j\left(q_{c 2}\right)$. Расчет энергии Гиббса с использованием аналитических выражений для сверхтекучих импульсов и параметров порядка, полученных в работе [9], подтверждает полученные численно результаты.

Величина $j_{s}$ уменьшается с ростом длины мостика и стремится к нулю при $L \rightarrow \infty$, поскольку при больших $L$ перераспределение импульсов $\Delta q=2 \pi / L$ мало, и солитоноподобное и синфазное состояния слабо отличаются друг от друга. Резкое падение разности энергий до нуля при малых токах для длины $L=15 \xi_{1}$ объясняется тем, что в области длин 10-20 $\xi_{1}$ при малых токах не существует устойчивых солитоноподобных состояний. При дальнейшем уменьшении длины мостика солитоноподобных состояний не существует при всех токах. Кроме того, при достаточно больших длинах мостика становятся устойчивыми многосолитонные состояния, которые также становятся энергетически более выгодными, чем синфазные и односолитонные, при токах $j_{s}<j<j\left(q_{c 2}\right)$.

Таким образом, при токе $j>j_{s}$ синфазное состояние становится энергетически невыгодным, т.е. метастабильным, что позволяет системе флуктуационным образом переходить в солитоноподобные состояния. Это может сказаться на $\delta F_{\text {thr }}(I)$, поскольку переход с проскальзыванием фазы между синфазным и солитоноподобным состоянием требует меньше энергии, чем между только синфазными. Используя описанную в разделе 2 методику, были численно построены токовые зависимости $\delta F_{\mathrm{thr}}(j)$ для различных длин мостика (рис. 3). Пороговая энергия образования ЦПФ в мостиках с длиной $L \lesssim \xi_{1}$, при которой солитоноподобные состояния отсутствуют, не проявляет каких-либо особенностей. Поскольку система находится в синфазном состоянии, в этом случае $\delta F_{\text {thr }}$ определяется суммой седловых состояний обеих

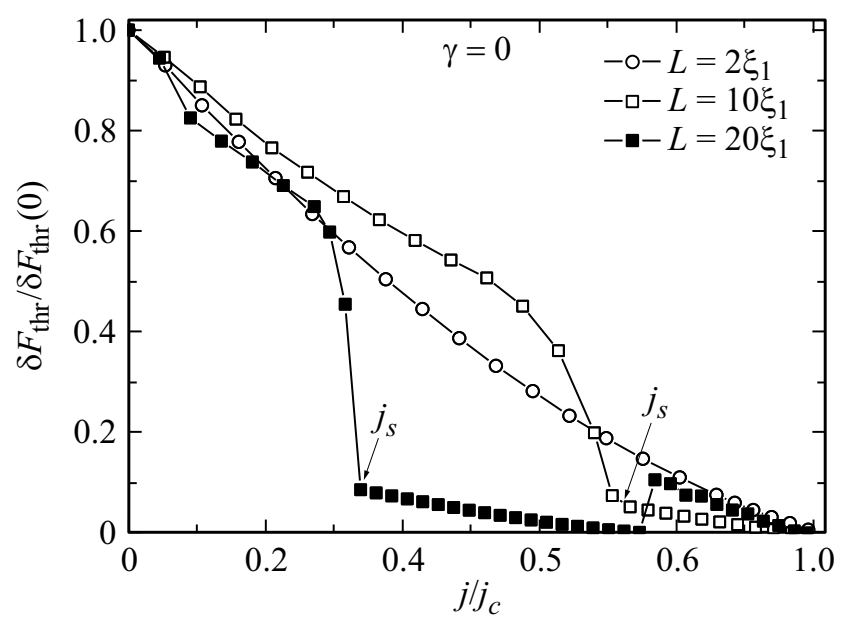

Рис. 3. Токовая зависимость пороговой энергии образования ЦПФ $\delta F_{\text {thr }}(j)$ при различных длинах мостика $L$ в случае нулевой межзонной джозефсоновской связи $\gamma=0$. 
зон $\delta F_{1 \text { saddle }}\left(j_{1}\right)+\delta F_{2 \text { saddle }}\left(j_{2}\right)$ и, аналогично однозонному случаю [3], с уменьшением длины $\delta F_{\text {thr }}(j)$ стремится к виду, приближенно описываемому выражением $\delta F(0)\left(1-j / j_{c}\right)^{3 / 2}$.

С ростом длины, когда становятся устойчивыми солитоноподобные состояния, зависимость $\delta F_{\text {thr }}(j)$ приобретает более сложный вид. При этом можно выделить несколько областей токов. При токах $j<j_{s}$ пороговая энергия образования ЦПФ определяется либо суммой седловых состояний обеих зон (при близких к нулевому токах либо при достаточно малых длинах, чтобы солитоноподобные состояния были устойчивы лишь при токах, близких к критическому), либо седловым состоянием сильной зоны. Сами состояния определяются условиями равенства набега фаз в обеих зонах $\Delta \theta_{1}=\Delta \theta_{2}$ и равенства суммы сверхтоков обеих зон заданному полному току $j=j_{1}+j_{2}$. Используя эти условия и полученное в работе [2] выражение для параметра порядка в седловом состоянии, в случае больших длин $\delta F_{\mathrm{thr}}(j)$ для этой области токов была найдена аналитически, и результат хорошо согласуется с проведенными расчетами. Несмотря на отличия от классической для длинных мостиков формы токовой зависимости, в области токов $j<j_{s}$ пороговая энергия образования ЦПФ, аналогично однозонному случаю, характеризует только вероятность переключения в резистивное состояние.

При токе $j>j_{s}$ становится возможным переключение в солитоноподобное состояние по механизму, при котором соответствующий переход происходит через проскальзывание фазы в слабой зоне. Поскольку образование ЦПФ только в слабой зоне требует гораздо меньше энергии, чем в сильной, вблизи $j_{s}$ пороговая энергия $\delta F_{\mathrm{thr}}(j)$ быстро спадает. При $j>j_{s}$ пороговая энергия образования ЦПФ определяется седловым состоянием слабой зоны, удовлетворяющим описанным выше условиям. В этой области токов $\delta F_{\text {thr }}(j)$ определяет вероятность перехода из синфазного состояния в состояние с одним солитоном и стремится к нулю при $j \rightarrow j\left(q_{c 2}\right)$. Таким образом, даже ниже $j\left(q_{c 2}\right)$ система может с довольно высокой вероятностью находиться в солитоноподобном состоянии благодаря флуктуационному механизму образования ЦПФ. При достаточно больших длинах этот переход может происходить при небольших средних токах переключения, которые не вызовут перегрева мостика. Поскольку в системе уже будут присутствовать фазовые солитоны, при $j\left(q_{c 2}\right)$ не возникнет проблемы, состоящей в том, что индуцированное током проскальзывание фазы может разогреть мостик и переключить его в резистивное состояние вместо солитонного, что устранит влияние присутствия солитонов в мостике на его критический ток, рассмотренное в работе [9].

В случае длин, при которых многосолитонные состояния неустойчивы, при токе $j\left(q_{c 2}\right)$ в зависимости $\delta F_{\mathrm{thr}}(j)$ наблюдается скачок, связанный с тем, что синфазное состояние становится неустойчивым, и $\delta F_{\mathrm{thr}}(j)$ в области токов $j>j\left(q_{c 2}\right)$ определяет энергию образования ЦПФ в ставшем основным состоянии с одним солитоном. В этом случае $\delta F_{\text {thr }}(j)$ стремится к нулю при стремлении тока к критическому для солитоноподобного состояния значению. Если длина мостика достаточно большая для образования многосолитонных состояний, то подобные скачки будут также происходить при токах, индуцирующих увеличение числа фазовых солитонов в мостике. Конечно, если учитывать возможность флуктуационного образования ЦПФ, то подобное флуктуационное изменение числа фазовых солитонов будет возможно и при меньших токах. Более того, при больших длинах мостика становится возможными переключение из синфазного в многосолитонное состояние через флуктуационный рост числа фазовых солитонов при $j<j\left(q_{c 2}\right)$.

\section{4. Флуктуационное образование фазовых солитонов в системах сконечной межзонной связью}

Рассмотрим влияние наличия конечной межзонной джозефсоновской связи $\gamma$ на $\delta F_{\mathrm{thr}}(j)$. В этом случае проскальзывания фазы могут приводить к возникновению состояний с нелинейным распределением $\theta(x)$ в виде солитонов, что также приводит к вариации амплитуды параметров порядка. В работе [9] было получено, что токовое образование фазовых солитонов возможно при малых значениях $\gamma$, меньших определенного значения (для использованных в расчетах параметров оно равно $\left.\gamma_{c}=0.022\right)$, а для больших $\gamma$ при всех токах сохраняется синфазное состояние. На рис. 4 представлены результаты расчетов $\delta F_{\text {thr }}(j)$ для $\gamma$ из этих двух областей. Для малых $\gamma<\gamma_{c}$ поведение токовой зависимости пороговой энергии качественно аналогично случаю $\gamma=0$. Здесь также есть ток $j_{s}$, при котором синфазное состояние становится метастабильным. Однако, межзонная связь

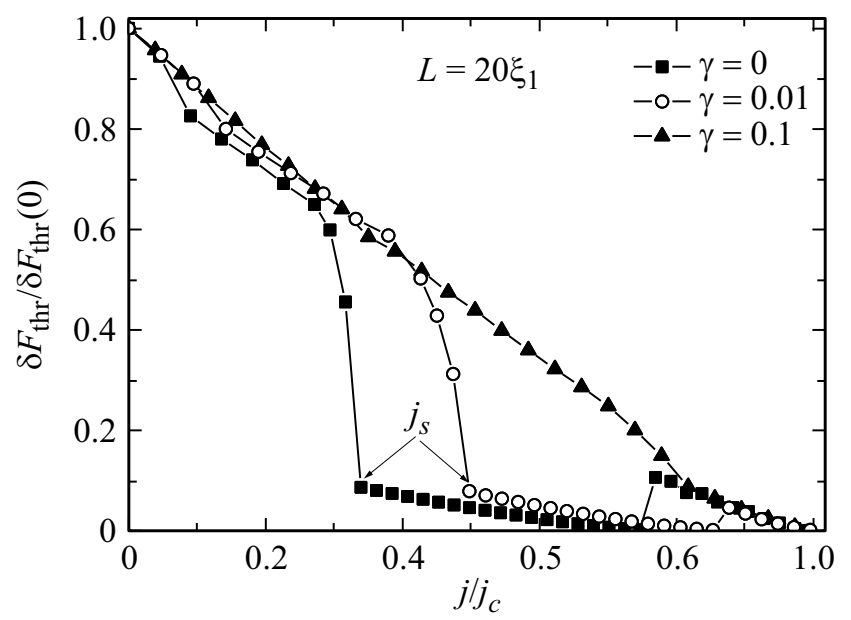

Рис. 4. Токовая зависимость пороговой энергии образования ЦПФ $\delta F_{\mathrm{thr}}(j)$ для мостика с длиной $L=20 \xi_{1}$ и значении параметра $R=j_{c 2} / j_{c 1}=0.15$ при различной силе межзонного джозефсоновского взаимодействия $\gamma$. 


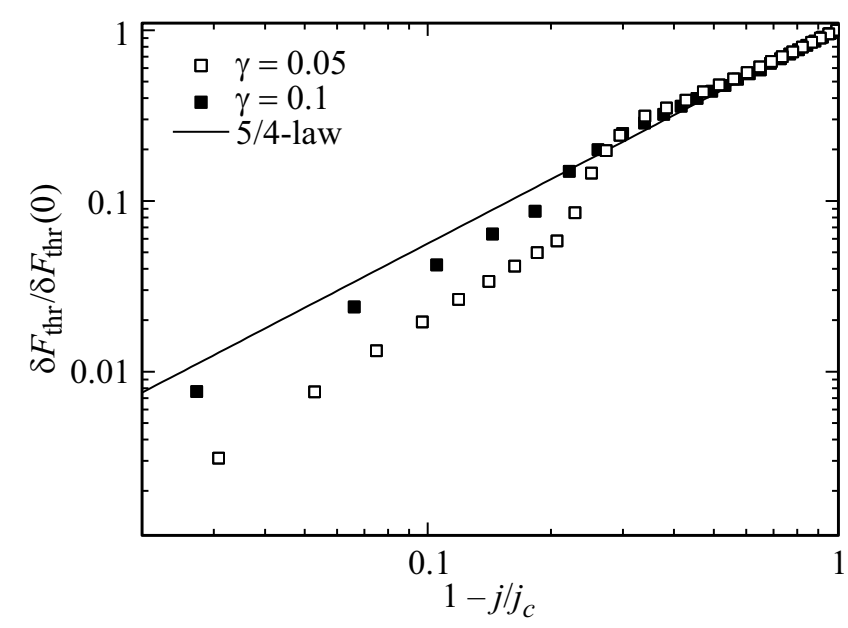

Рис. 5. Сравнение токовой зависимости пороговой энергии образования ЦПФ $\delta F_{\mathrm{thr}}(j)$ при различной силе межзонного джозефсоновского взаимодействия $\gamma \mathrm{c}$ аппроксимирующей зависимостью для однозонного случая вида $\delta F(0)\left(1-j / j_{c}\right)^{5 / 4}$. Данные представлены в логарифмическом масштабе.

увеличивает энергию солитонного состояния, и поэтому $j_{s}$ растет с ростом $\gamma$. Соответственно, падение пороговой энергии $\delta F_{\text {thr }}$ и флуктуационное образование фазовых солитонов происходит при больших токах. Начиная с $\gamma_{c}$, солитонные состояния возможны, но их энергия в той области токов, где они могут существовать, всегда выше синфазных состояний. Как и в случае индуцированных током проскальзываний фаз, межзонная джзефсоновская связь оказывается достаточно сильной, чтобы подавить независимое образование ЦПФ в разных зонах. Поэтому, хотя при больших токах ЦПФ образуется также прежде всего в слабой зоне, наличие связи приводит также к возникновению ЦПФ в сильной зоне, и флуктуационное образование солитонов при больших $\gamma$ отсутствует. При этом, благодаря межзонной связи, падение $\delta F_{\text {thr }}(j)$ становится все менее резким с ростом $\gamma$, и, как видно из рис. 5, в целом с ростом силы межзонной связи токовая зависимость для длинных мостиков стремится к характерному для однозонного случая виду $\delta F(0)\left(1-j / j_{c}\right)^{5 / 4}$, полученному в качестве аппроксимации результата Лангера и Амбегаокара в [17]. Это связано с тем, что двузонные сверхпроводники с сильной межзонной связью эффективно могут описываться одним параметром порядка.

\section{5. Заключение}

В настоящей работе на основе теории ГинзбургаЛандау была рассчитана токовая зависимость пороговой энергии образования ЦПФ $\delta F_{\mathrm{thr}}(j)$ для двузонного сверхпроводящего мостика. Обнаружено, что для случая слабой межзонной джозефсоновской связи возможно флуктуационное образование фазовых солитонов при токах выше определенного значения $j_{s}$ (растущего с уменьшением длины мостика и увеличением силы межзонного взаимодействия $\gamma$ ), при котором синфазное состояние становится метастабильным. Это сопровождается резким падением $\delta F_{\mathrm{thr}}$ в области токов вблизи $j_{s}$, которое сглаживается с ростом силы межзонной связи $\gamma$. При $\gamma>\gamma_{c}$ флуктуационное образование фазовых солитонов, как и в случае формирования солитонов током, подавляется, и при больших значениях $\gamma \gg \gamma_{c}$ токовая зависимость $\delta F_{\text {thr }}$ может быть аппроксимирована выражением $\delta F(0)\left(1-j / j_{c}\right)^{5 / 4}$ в широкой области токов, аналогично однозонному случаю.

\section{Список литературы}

[1] Б.И. Ивлев, Н.Б. Копнин. УФН 142, 435 (1984).

[2] J.S. Langer, V. Ambegaokar. Phys. Rev. 164, 498 (1967).

[3] П.М. Марычев, Д.Ю. Водолазов. Письма в ЖЭТФ 103, 458 (2016).

[4] H.J. Choi, D. Roundy, H. Sun, M.L. Cohen, S.G. Louie. Nature 418, 758 (2002).

[5] Y. Tanaka. Phys. Rev. Lett. 88, 017002 (2001).

[6] H. Bluhm, N.C. Koshnick, M.E. Huber, K.A. Moler. Phys. Rev. Lett. 97, 237002 (2006).

[7] Y. Tanaka, H. Yamamori, T. Yanagisawa, T. Nishio, S. Arisawa. Physica C 548, 44 (2018).

[8] A. Gurevich, V.M. Vinokur. Phys. Rev. Lett. 97, 137003 (2006).

[9] P.M. Marychev, D.Yu. Vodolazov. Phys. Rev. B 97, 104505 (2018).

[10] N. Kopnin. Theory of Nonequilibrium Superconductivity. Oxford University Press, N. Y. (2001) 342 p.

[11] A. Gurevich, V.M. Vinokur. Phys. Rev. Lett. 90, 047004 (2003).

[12] M.E. Zhitomirsky, V.H. Dao. Phys. Rev. B 69, 054508 (2004).

[13] A. Gurevich. Phys. Rev. B 67, 184515 (2003).

[14] David Pekker, Alexey Bezryadin, David S. Hopkins, Paul M. Goldbart. Phys. Rev. B 72, 104517 (2005).

[15] D.Yu. Vodolazov. Phys. Rev. B 85, 174507 (2012).

[16] N.W. Ashcroft. Phys. Rev. Lett. 21, 1748 (1968)

[17] M. Tinkham, J.U. Free, C.N. Lau, N. Markovic. Phys. Rev. B 68, 134515 (2003).

Редактор Ю.Э. Китаев 